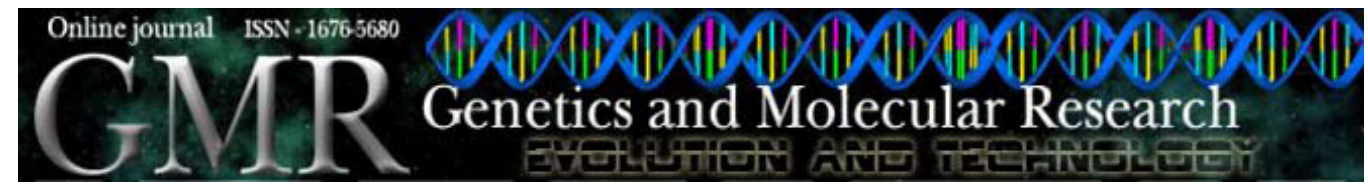

\title{
Simultaneous presence of bovine papillomavirus and bovine leukemia virus in different bovine tissues: in situ hybridization and cytogenetic analysis
}

\author{
A. Yaguiu', M.L.Z. Dagli ${ }^{3}$, E.H. Birgel Jr. ${ }^{4}$, B.C.A. Alves Reis ${ }^{5}$, \\ O.P. Ferraz ${ }^{1}$, E.M. Pituco ${ }^{6}$, A.C. Freitas ${ }^{2}$, W. Beçak ${ }^{1,2}$ and R.C. Stocco ${ }^{1}$ \\ ${ }^{1}$ Laboratório de Genética, Instituto Butantan, São Paulo, SP, Brasil \\ ${ }^{2}$ Departamento de Genética, UFPE, Recife, PE, Brasil \\ ${ }^{3}$ Departamento de Patologia, FMVZ-USP, São Paulo, SP, Brasil \\ ${ }^{4}$ Departamento de Clínica Médica, FMVZ-USP, São Paulo, SP, Brasil \\ ${ }^{5}$ Departamento de Imunologia, ICB-USP, São Paulo, SP, Brasil \\ ${ }^{6}$ Laboratório de Viroses de Bovídeos, Instituto Biológico, \\ São Paulo, SP, Brasil \\ Corresponding author: R.C. Stocco \\ E-mail: ritastocco@yahoo.com or ritastocco@butantan.gov.br
}

Genet. Mol. Res. 7 (2): 487-497 (2008)

Received March 31, 2008

Accepted May 12, 2008

Published June 10, 2008

\begin{abstract}
Bovine papillomavirus (BPV) DNA sequences were detected in different tissues, in addition to epithelium. Cytogenetic abnormalities were observed in blood lymphocytes. The presence of more than one virus in a single tissue is a difficult aspect to evaluate, especially when the DNA sequences are detected in tissues that are not specifically targeted by the virus. BPV and bovine leukemia virus (BLV) are clastogenic, causing chromosome aberrations in peripheral blood lymphocytes. In the present study, we investigated the simultaneous presence of DNA sequences of both viruses and
\end{abstract}


the possibility of vertical transmission and compared the types of chromosome aberrations related to viral action. BPV 1, 2, and 4 DNA sequences were found in three females of the herd and in their offspring. BLV DNA sequences were not detected in their progeny. A newborn calf that was negative for BLV infection showed specific chromosome rearrangements possibly related to the effect of infection with BPV.

Key words: Bovine papillomavirus; Bovine leukemia virus; Chromosome aberrations; Virus co-infection

\section{INTRODUCTION}

The simultaneous presence of two or more viral agents infecting a single individual in the same tissue, suggesting co-infection, has not often been described and obviously leads to serious consequences as to augmenting the difficulties of disease prophylaxis, control and therapeutics. Virus co-infection can involve the presence of different types of the same virus and/or different viruses related to different diseases. The co-existence of different pathogens in the same tissue can result in deleterious interactions with the host cells and with the host chromatin.

In bovine species, two specific viruses, which are endemic, are capable of interacting with the host chromatin, namely, bovine papillomavirus (BPV) and bovine leukemia virus (BLV) (Castro et al., 1988; Stocco dos Santos et al., 1998; Leal et al, 2003). BPV is an important etiologic agent of tumors in cattle (mostly of the upper alimentary tract and bladder), causing dramatic economic losses (Campo et al., 1992, 1994; Campo, 2003; Carvalho et al., 2003; Freitas et al., 2003). On the other hand, BLV, a B-lymphocyte-tropic retrovirus, causes enzootic bovine leukosis that can progress to lymphoma. While vertical transmission of BPV has already been described (Stocco dos Santos et al., 1998; Freitas et al., 2003), there are few reports about BLV transmission to offspring.

Infections by papillomaviruses lead to hyperplastic lesions, such as warts, papillomas or condylomas, in several species including humans (Campo, 2003). These lesions are usually benign, but environmental co-factors, such as consumption of the fern Pteridium aquilinum $v$. aracnoideum, have been described as inducing cell modifications that result in neoplastic transformation (Recouso et al., 2003). Natural regression of papillomas can occur and the histological examination of the lesions reveals an intense infiltration of lymphocytes in the dermis and epithelium.

Even though BPV is described as being epithelium-specific, we have previously reported the presence of virus DNA sequences in different cells such as those of peripheral blood and gametes (Stocco dos Santos et al., 1998; Carvalho et al., 2003; Freitas et al., 2003). The significance of the presence of these nucleic acid sequences in these tissues has not yet been determined, considering that there is yet no evidence of expression of these nucleic acid sequences and their potential to initiate infection.

There are few cytogenetic studies about BPV lesions that can be found in the literature. Amongst these studies, we have described chromosomal abnormalities in peripheral blood lymphocytes collected from chronically infected animals (Moura et al., 1988), from 
animals experimentally exposed to the virus (Stocco dos Santos et al., 1998) and in a cell line obtained from bovine palate fibroblasts submitted to a specific protocol of transfection with active Ha-ras, BPV-4 protein E7 and exposed to a quercetin pulse (Leal et al., 2003). In all these situations, we have described a significant increased rate of chromosomal aberrations, with peculiar rearrangements possibly originating from centric fusions. These chromosome aberrations detected in cells showing BPV DNA sequences suggest that these sequences act on the host chromatin and therefore could be expressed.

While BPV has double-stranded DNA, BLV is an exogenous retrovirus (Lee et al., 2005). In BLV infection, abnormal regulation of B cell development results in sustained non-malignant polyclonal expansion of $\mathrm{CD}^{+} \mathrm{B}$ cells, referred to as persistent lymphocytosis which also occurs in bovine lymphosarcoma with variable progression of the disease (Castro et al., 1988). Nonrandom chromosomal abnormalities and an increased rate of chromosome aberrations were described in peripheral lymphocytes of animals affected with persistent lymphocytosis (Castro et al., 1988). The early events that result in infection by BLV (and its human analog, human T-cell leukemia virus) remain to be elucidated. Allogenic cells containing viral nucleic acid create an entrance for the viruses into naive individuals via blood, semen, or breast milk and can produce viruses that continue to infect cells of the new host, being detectable in the blood one week after infection (Fulton Jr., 2006). The preferred host cells of BLV are B lymphocytes (Kenyon and Piper, 1977), and the disease related to this infection is described as occurring only in cattle, which can be silent in that it does not show symptoms. However, $30 \%$ of infected cattle can develop persistent lymphocytosis, which may progress to lymphoid tumors (Fulton Jr. et al., 2006). BLV has been reported to infect human cells in vitro and cause tumors and erythroleukemia in primates (McClure et al., 1974; Johnson et al., 1994b; Lee et al., 2005). Increased rates of myeloid leukemia and non-Hodgkin's lymphomas were detected among workers in facilities that deal with meat processing, suggesting that BLV is potentially oncogenic in humans (Pearce et al., 1986; Johnson, 1994a).

The aim of the present study was to analyze the simultaneous presence of BPV and BLV DNA sequences in blood samples obtained from bovine females and their newborn calves and the eventual vertical transmission of the viruses. The detection of BPV DNA sequences in other tissues, apart from blood, would be confirmed by polymerase chain reaction (PCR) and/or in situ hybridization. A cytogenetic evaluation was carried out on lymphocytes obtained from bovine females and their progeny.

\section{MATERIAL AND METHODS}

\section{Animal selection}

We selected 3 cows (Bos taurus taurus) with 3 years old, presenting different symptoms relating to BPV and BLV infection, and high levels of lymphocytosis: animal 1: Female presenting cutaneous papillomatosis; animal 2: Female presenting enzootic hematuria; animal 3: Female without clinical symptoms of BPV infection.

These animals came from a herd with a high prevalence of papillomatosis and bovine leukemia. The animals were evaluated just before delivering their offspring, and samples were also collected from the three newborn male offspring upon their delivery. 


\section{Sample collection}

Blood and tissue samples were collected for analysis. A sample of $10 \mathrm{~mL}$ blood was collected for each analysis using disposable syringes and needles and stored in tubes containing an anticoagulant (EDTA for molecular analysis and heparin for cytogenetic procedures). Wart samples were obtained by parallel incision to the surface of the skin using a disposable sterile scalpel. Samples of placenta, uterus and amniotic liquid were also collected immediately after delivery and kept at $4^{\circ} \mathrm{C}$ until the procedures.

\section{DNA extraction}

All samples collected were submitted to total DNA extraction using a commercially available kit Qiagen DNA easy Blood and Tissue kit (Qiagen), according to manufacturer instructions.

\section{Detection of viral nucleic acid sequences}

We decided to investigate the presence of BPV types 1, 2 and 4 DNA sequences considering that these types of BPV were the most prevalent in the herd.

\section{BPV detection}

For the detection of BPV, $300 \mathrm{ng}$ DNA, isolated from the samples, were amplified by PCR using primers specific to BPV1 (Fw: 5'-ggagcgectgctaactatagga-3' and Rv: 5'-atctgttgtttgggtggtgac-3'), BPV2 (Fw: 5'-gttataccacccaaagaagaccct-3' and Rv: 5'-ctggttgcaacagctctctttctc-3') and BPV4 (Fw: 5'-gctgaccttccagtcttaat-3' and Rv: 5'-cagtttcaatctcctcttca-3'). These primers are complementary to the L2 and L1 regions of the BPV genomes, for BPV1 and BPV2, respectively, and to the E7 region of BPV4, amplifying fragments of 301, 164 and $170 \mathrm{bp}$, respectively. The PCR protocol was developed according to Stocco dos Santos et al. (1998) using appropriate controls. As a control of the quality of DNA for PCR, the bovine $\beta$-globin gene was amplified using specific primers (Fw: 5'-AAC CTC TTT GTT CAC AAC CAG-3' and Rev: 5'-CAG ATG CTT AAC CCA CTG AGC-3') that yielded a 430-bp fragment, according to a protocol previously described by Freitas et al. (2003).

\section{BLV detection}

The detection of BLV sequences was carried out using a nested-PCR technique. For the first amplification, we used a pair of primers spanning the entire env gene region that encodes for envelope glycoprotein gp51: 5' TGGAGATGCTCCCTGTCCCT 3' and 5' CTCCTACCCGGGTCAGACGT 3'. The second amplification reaction was carried out using an internal pair of primers [5' GTGCCAAGTCTCCCAGATACA 3' and 5' TATAGCACAGTCTGG 3'] yielding a 427-bp fragment.

The above described amplifications for BPV and BLV were performed in a PTC$100^{\mathrm{TM}}$ thermal cycler (MJ Research, Inc.) programmed as follows: an initial cycle of 3 
min at $94^{\circ} \mathrm{C}$, followed by 35 cycles of 1 min at $94^{\circ} \mathrm{C}, 1 \mathrm{~min}$ at $65^{\circ} \mathrm{C}$, and $2 \min$ at $72^{\circ} \mathrm{C}$, and a final step of $5 \mathrm{~min}$ at $72^{\circ} \mathrm{C}$. For BLV detection, the products of the first amplification were diluted 100-fold and then subjected to the second amplification, using similar conditions.

\section{Sequencing}

We selected BPV1 PCR products for sequencing to serve as positive controls of the BPV determination using wart samples and by sequencing cloned BPV1 PCR products using the TOPO TA Cloning ${ }^{\mathbb{B}}$ kit for cloning. The cloned fragments were sequenced using an A.L.F. express DNA Sequencer (Pharmacia Biotech ${ }^{\circledR}$ ). Both strands of two selected clones from each sample were sequenced using Thermo Sequenase Fluorescent Labelled Primer Cycle Sequencing kit with 7-deaza-dGTP, with a universal vector and reverse primers.

\section{Cytogenetic analysis}

In order to verify the presence of chromosomal rearrangements, cytogenetic studies were carried out using short-term peripheral lymphocyte cultures of collected samples corresponding to those used in molecular analysis, according to previously described protocols (Stocco dos Santos et al., 1998), including the C banding procedure according to Leal et al. (2003).

\section{In situ hybridization}

Wart and uterus samples were prepared onto histological slides bearing tissue sections $(1.5 \mathrm{~mm})$ that were stained with hematoxylin and eosin by routine procedures and submitted to in situ hybridization, which was developed using biotin-labeled PCR products as probes. The probes were labeled using a DAKO GenPoint kit, following the protocol suggested for histological slides. Negative control slides were prepared using samples obtained from the infected animals, and were submitted to the same procedure but omitting the use of probes.

\section{RESULTS}

Using the $\beta$-globin gene as a control of DNA sample quality, the PCR products allowed us to verify that all samples collected were appropriate for further amplification.

The PCR analysis showed that different types of BPV sequences were present in blood samples collected from bovine females as well as from their offspring. Two females had BPV DNA sequences of types 2 and 4 (animals 1 and 3) and the other female showed types 1, 2 and 4 (animal 2), in blood samples. Two newborn males showed DNA sequences of the three types of BPV (1,2 and 4) in blood samples, as illustrated in Figures 1, 2 and 3 (the offspring of animal 1). On the other hand, the nested-PCR procedure detected the presence of BLV sequences in the blood samples of two of the females evaluated, namely, animal 1 and animal 3, which is also illustrated in Figure 4. 


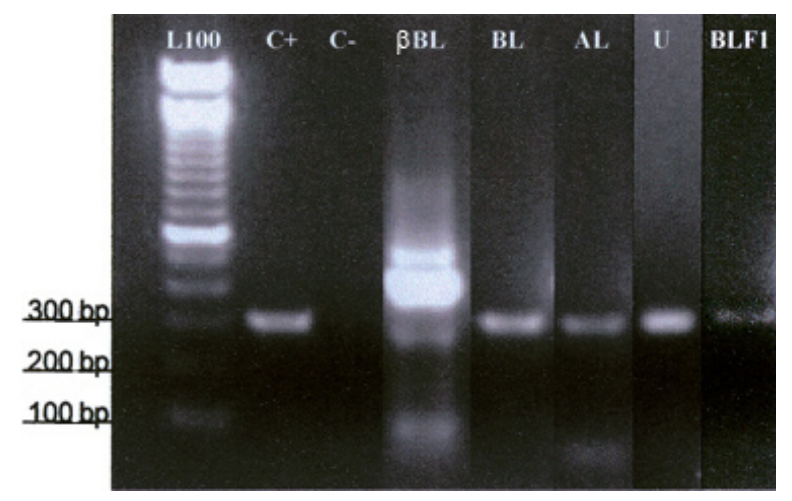

Figure 1. Bovine papillomavirus type 1: DNA sequence detection by PCR. Agarose gel electrophoresis: lane L100: 100-bp ladder; lane $C+$; positive control; lane $C$-: negative control; lane $\beta B L$ : $\beta$-globin; lane $B L$ : blood sample; lane $A L$ : amniotic fluid; lane $U$ : uterus; lane BLF1: blood sample of offspring born to female 1.

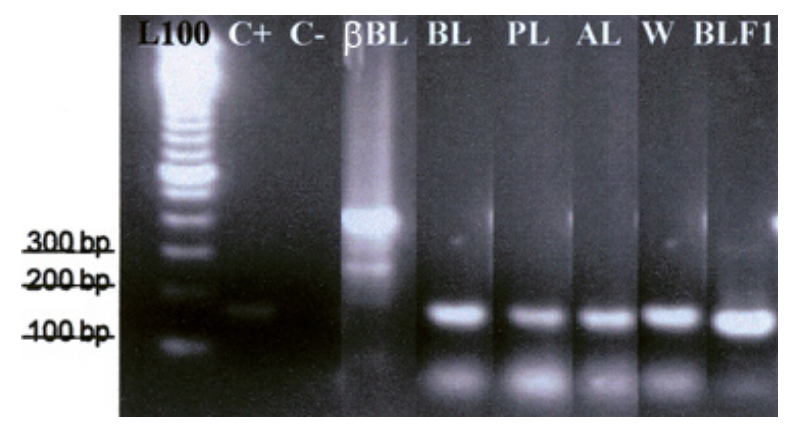

Figure 2. Bovine papillomavirus type 2: DNA sequence detection by PCR. Agarose gel electrophoresis: lane L100: 100-bp ladder; lane $C+$ : positive control; lane $C$-: negative control; lane $\beta B L$ : $\beta$-globin; lane $B L$ : blood sample; lane PL: placenta sample; lane $A L$ : amniotic fluid; lane $W$ : wart sample; lane $B L F 1$ : blood sample of offspring born to female 1 .

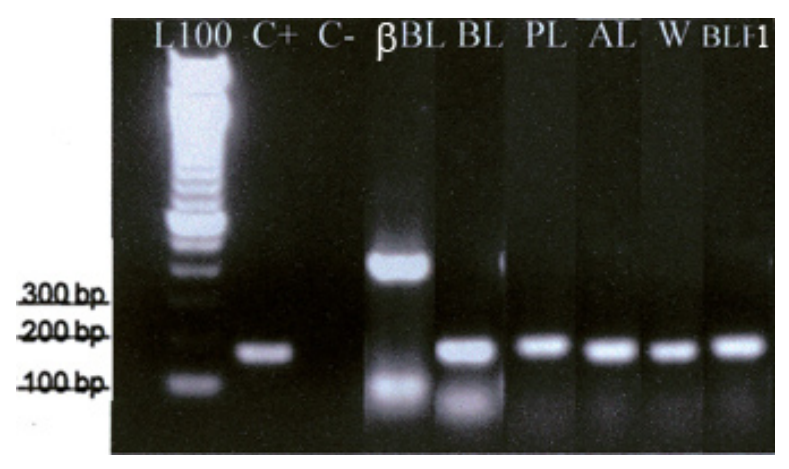

Figure 3. Bovine papillomavirus type 4: DNA sequence detection by PCR. Agarose gel electrophoresis: lane L100: 100-bp ladder; lane $C+$ : positive control; lane $C$-: negative control; lane $\beta B L$ : $\beta$-globin; lane $B L$ : blood sample; lane $P L$ : placenta sample; lane $A L$ : amniotic fluid; lane $W$ : wart sample; lane BLF1: blood sample of offspring born to female 1. 


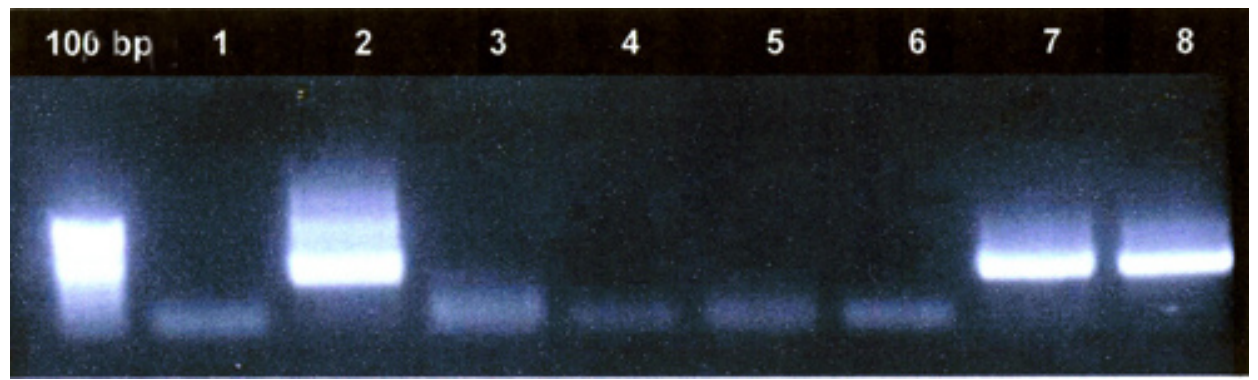

Figure 4. Bovine leukemia virus detection by nested-PCR. Agarose gel electrophoresis. Lane 100 bp: 100-bp ladder; lane 1: animal 2 amniotic liquid; lane 2: animal 1 blood sample; lane 3: animal 3 uterus sample; lane 4: negative control; lane 5: animal 3 amniotic fluid; lane 6: offspring born to animal 1 blood sample; lane 7: animal 3 blood sample; lane 8: positive control.

Samples of wart, uterus, amniotic fluid, and placenta from the three females were collected and analyzed for the presence of BPV DNA sequences. The PCR products showed the presence of DNA sequences of the three types of BPV investigated (1,2 and 4) in the wart sample collected from one of the females (animal 1), and in the amniotic fluid samples obtained from all three females (animals 1,2 and 3). The uterus fragments collected from two of the females (animals 2 and 3 ) contained DNA sequences of the three types of BPV investigated (1,2 and 4). The placental samples collected from animals 1,2 and 3 were positive for the presence of DNA sequences of BPV types 2 and 4.

The blood samples obtained from animal 1 F1 offspring and animal 2 F1 offspring were positive for BPV1, BPV2 and BPV4. These data are summarized in Table 1.

Table 1. Results of virus sequence detection.

\begin{tabular}{|c|c|c|c|c|c|c|}
\hline Animal & Sample & BPV1 & BPV2 & BPV4 & $\%$ BOV1 homology & BLV \\
\hline \multirow[t]{5}{*}{1 ㅇ } & blood & - & + & + & NV & + \\
\hline & amniotic fluid & + & + & + & NV & \\
\hline & placenta & - & + & + & NV & \\
\hline & wart & + & + & + & NV & \\
\hline & blood F1 (ふ) & + & + & + & NV & \\
\hline \multirow[t]{5}{*}{$2 q$} & blood & + & + & + & NV & \\
\hline & amniotic fluid & + & + & + & 99 & \\
\hline & placenta & - & + & + & $\mathrm{NV}$ & \\
\hline & uterus & + & + & + & 98 & \\
\hline & blood F1 $(ð)$ & + & + & + & NV & \\
\hline \multirow[t]{5}{*}{$3 q$} & blood & - & + & + & NV & + \\
\hline & amniotic fluid & + & + & + & 99 & \\
\hline & placenta & - & + & + & NV & \\
\hline & uterus & + & + & + & 98 & \\
\hline & blood F1 $(\precsim)$ & - & - & - & NV & \\
\hline
\end{tabular}

BVP1, 2, 4 = bovine papillomavirus types 1,2 and 4, respectively; $\mathrm{BVL}=$ bovine leukemia virus; $\mathrm{NV}=$ not verified; - = negative; + = positive; F1 = offspring born to animal 1.

In order to perform the in situ procedures, the BPV1 PCR products from amniotic fluid and uterus fragments collected from two of the females (animals 2 and 3) were previously sequenced and were used as positive control for the PCR products. The sequencing results 
showed a high homology of the samples with the L1 region of BPV1 (Table 1).

The tissue samples were prepared for histology and for in situ detection of BPV DNA. The wart sample cross-section consisted of homogeneous connective tissue, covered by hyperplastic epidermis with hyperkeratosis. In situ hybridization with labeled PCR products revealed the presence of the three BPV types investigated: 1, 2 and 4 (Figure 5). Histological analysis of the uterus fragments showed characteristics of post-delivery tissue such as hyperplastic musculature and cavernous hemangiomas. In situ hybridization with labeled PCR products revealed the presence of the three BPV types investigated: 1, 2, and 4, more specifically in the blood cells found in the hemangiomas (Figure 6).

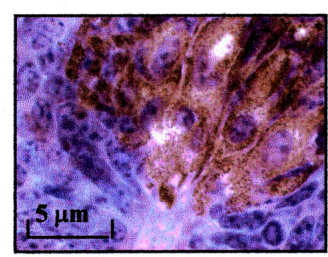

BPV1

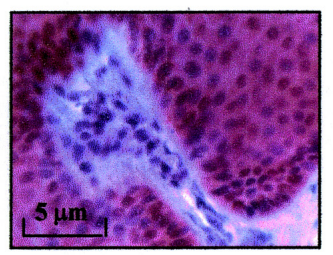

BPV2

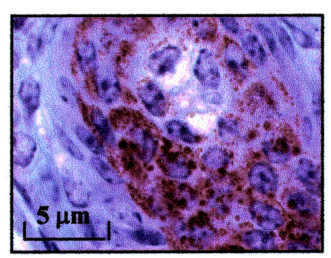

BPV4

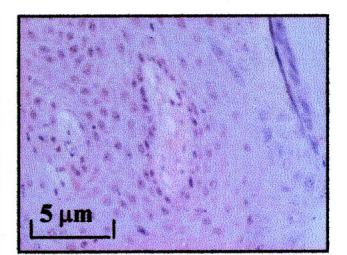

Negative control

Figure 5. Bovine papillomavirus locations in wart sections by in situ hybridization procedure. BVP1, 2, 4= bovine papillomavirus types 1,2 , and 4 , respectively.

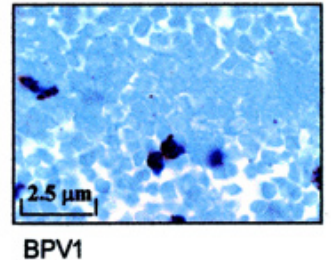

BPV1

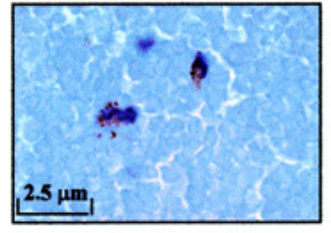

BPV2

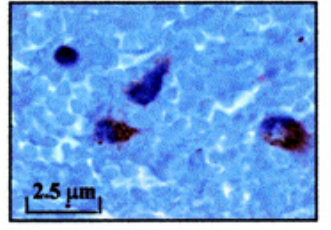

BPV4

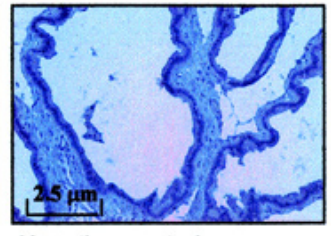

Negative control

Figure 6. Bovine papillomavirus locations in uterus sections by in situ hybridization procedure. BVP1, 2, $4=$ bovine papillomavirus types 1,2 , and 4 , respectively.

Cytogenetic analysis was performed in short-term peripheral lymphocyte cultures of blood samples collected from the three females and their offspring. Considering that BPV and BLV are clastogenic, the metaphases were analyzed using conventional Giemsa staining and C banding for the detection of aberrations and/or rearrangements which could eventually be related to only one virus type. Our analysis detected chromosomal aberrations in all samples, including breaks, acentric fragments and chromosome markers. These chromosomal markers could easily be identified, since they were metacentric, while in a normal bovine karyotype, the autosomes are acrocentric and only the $\mathrm{X}$ and $\mathrm{Y}$ chromosomes are submetacentric in Bos taurus.

$\mathrm{C}$ banding showed centric associations and fusions. Since our purpose was to distinguish aberrations related to a specific virus type, we directed our attention to the blood sample collected from the calf born to female 2. This female did not show BLV in its blood sample, and its F1 offspring was also negative for the virus in its blood sample. The metaphases in tissues obtained from the animals studied and the types of chromosomal aberrations detected 
are shown in Figure 7. Centric fusion and telomere associations, possibly related to BPV, were present in all samples of the female $2 \mathrm{~F} 1$ offspring.

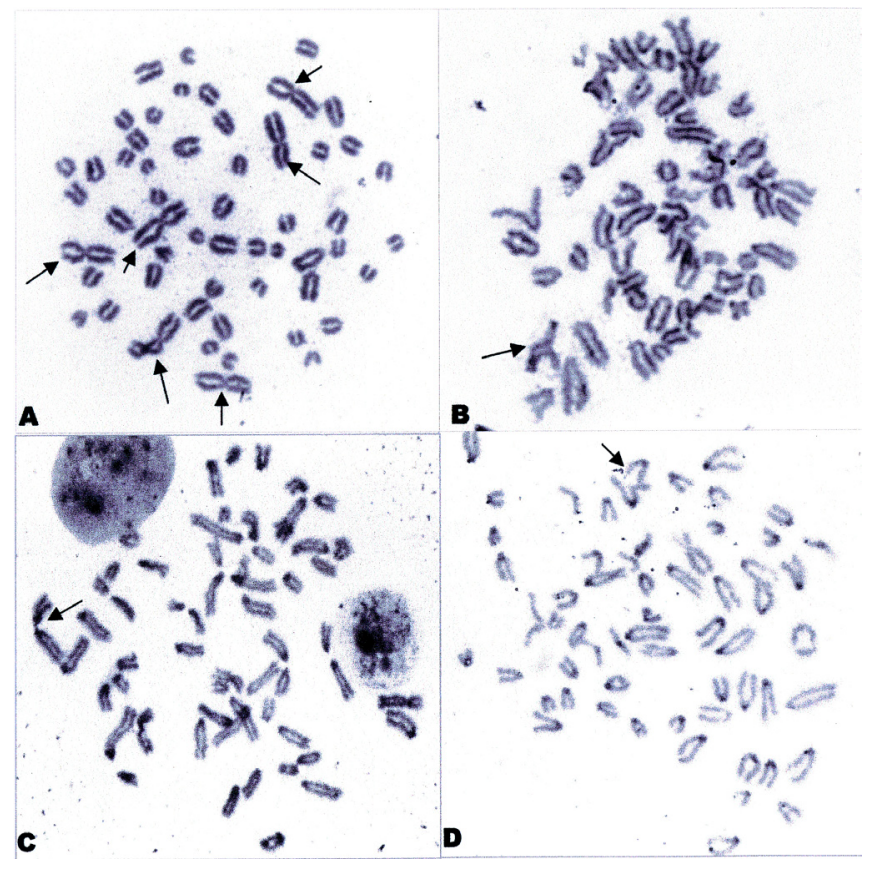

Figure 7. Peripheral lymphocyte metaphases obtained by short-term cultures and stained with Giemsa. The arrows indicate chromosome rearrangements. In $\mathrm{A}$ and $\mathrm{C}$, the arrows indicate centric fusions. In $\mathrm{B}$ and $\mathrm{D}$, the arrows indicate telomeric rearrangements. $\mathrm{A}, \mathrm{B}$ and $\mathrm{C}$ were obtained from animals showing BPV and BLV sequences. D was obtained from an animal that was BPV-positive and BLV-negative.

\section{DISCUSSION}

The papillomavirus infection starts in the basal layers of the epithelium and its progress occurs throughout the activation of $\mathrm{E}$ (early) and L (late) genes, producing virions on the external layers of the tissue (Freitas et al., 2003). The entrance of a non-packed virus in a target cell occurs in two steps (Campo, 1995): 1) attachment, interaction with the cell membrane through the binding to a surface receptor molecule; 2) penetration of the viral particle. This corroborates the hypothesis that papillomaviruses attach to a conserved receptor abundantly present on the cell surface.

Recent studies have discussed several pathways for the entrance of the papillomavirus into the cell, using different molecules at the cell surface as receptors, such as integrin $\alpha 6 \beta 4$ or $\alpha 6 \beta 1$, heparan sulfate or, alternatively, other mechanisms such as endocytosis via a clathrindependent pathway (Freitas et al., 2003).

The present report is the by-product of a pilot study concerning BPV co-infection with $\mathrm{BLV}$, detection of virus sequences in non-specific target cells and interaction with host chromatin. 
In order to evaluate the specific action of BPV on host chromatin, we investigated the eventual presence of BLV nucleic acid sequences in the animals studied, considering that both viruses have already been described as demonstrating clastogenic effects in peripheral lymphocytes and fibroblasts (Castro et al., 1988; Stocco dos Santos et al., 1998; Leal et al., 2003). Not only were sequences of both viruses, BPV and BLV, found in some of the animals studied, but we also detected clastogenic effects in both the presence and absence of BLV.

These results demonstrate the simultaneous presence of three BPV types, namely, 1, 2 and 4, not only in their target cells, wart epithelium cells, but also in blood. BPV sequences were also detected in uterus, amniotic fluid and placenta. Even though the expression of BPV in cells that are not targeted by the virus is controversial and even questionable, some data presented here suggest that these sequences could be active. DNA sequences of the three BPV types detected in the wart samples were also detected in the blood and reproductive tissue samples. Moreover, BPV sequences were found in blood samples collected from the bovine offspring, indicating the occurrence of vertical transmission of BPV. Considering the distribution of the DNA sequences, we can argue that the transmission of the virus occurred either via blood cells present in reproductive tissues or directly by reproductive cells. In the case of female 1, her blood sample was negative for BPV1, and yet her offspring calf tested positive for BPV1 DNA sequences in its blood sample, probably a result of infection from its mother's amniotic liquid, which was positive for BPV1. The same did not occur with female 3, since her offspring did not show BPV sequences, in spite of the fact that the three BPV types were detected in all samples obtained from the females.

Chromosome aberrations and centric fusions, similar to those previously described related to BPV oncoprotein action (Leal et al., 2003), were identified in all samples, suggesting that BPV has an effect on host chromatin, which could mean that the nucleic acid sequences detected could be active and eventually be transmitted to the offspring. The rearrangements found in the cells of the offspring born to the female not infected by BLV suggest that these alterations are caused by BPV.

The high incidence of the diseases caused by BPV and BLV could be due to the possibility of co-infection with different types of BPV and BLV and the possible expression of these viruses in other tissues that are not their preferred targets. All these data must be taken into consideration when applying vaccines against these viruses.

It thus seems that the eventual interaction of these two different viruses, whose modes of transmission are yet poorly understood, could lead to an enhancement of mechanisms of their transmission and expression.

\section{ACKNOWLEDGMENTS}

The authors would like to thank Carolina da Paz Sabino for her editorial support, CNPq and FAPESP for the fellowships (project Nos. 507376/2004-8, 554816/2006-7 and 2006/02439-6, respectively). We thank John McCulloch for reviewing the manuscript.

\section{REFERENCES}

Campo MS (1995). Infection by bovine papillomavirus and prospects for vaccination. Trends Microbiol. 3: 92-97. Campo MS (2003). Papillomavirus and disease in humans and animals. Vet. Comp. Oncol. 1: 3-14.

Campo MS, Jarrett WF, Barron R, O’Neil BW, et al. (1992). Association of bovine papillomavirus type 2 and bracken fern 
with bladder cancer in cattle. Cancer Res. 52: 6898-6904.

Campo MS, Jarrett WF, O'Neil W and Barron RJ (1994). Latent papillomavirus infection in cattle. Res. Vet. Sci. 56: 151-157.

Carvalho C, Freitas AC, Yaguiu A, Góes LGB, et al. (2003). Bovine papillomavirus type 2 in reproductive tract and gametes of slaughtered bovine females. Braz. J. Microbiol. 34: 82-84.

Castro NH, Walter J, dos Santos RC, D’Angelino JL, et al. (1988). Cytogenetic study of cattle affected by persistent lymphocytosis. Zentralbl. Veterinarmed. A 35: 380-384.

Freitas AC, Carvalho C, Brunner O, Birgel EH Jr, et al. (2003). Viral DNA sequences in peripheral blood and vertical transmission of the virus: a discussion about BPV-1. Braz. J. Microbiol. 34: 76-78.

Fulton BE Jr, Portela M and Radke K (2006). Dissemination of bovine leukemia virus-infected cells from a newly infected sheep lymph node. J. Virol. 80: 7873-7884.

Johnson ES (1994a). Cancer mortality among workers in the meat department of supermarkets. Occup. Environ. Med. 51: 541-547.

Johnson ES (1994b). Poultry oncogenic retroviruses and humans. Cancer Detect. Prev. 18: 9-30.

Kenyon SJ and Piper CE (1977). Cellular basis of persistent lymphocytosis in cattle infected with bovine leukemia virus. Infect Immun. 16: 891-897.

Leal AM, Ferraz OP, Carvalho C, Freitas AC, et al. (2003). Quercetin induces structural chromosome aberrations and uncommon rearrangements in bovine cells transformed by the E7 protein of bovine papillomavirus type 4 . Vet. Comp. Oncol. 1: 15-21.

Lee J, Kim Y, Kang CS, Cho DH, et al. (2005). Investigation of the bovine leukemia virus proviral DNA in human leukemias and lung cancers in Korea. J. Korean Med. Sci. 20: 603-606.

McClure HM, Keeling ME, Custer RP, Marshak RR, et al. (1974). Erythroleukemia in two infant chimpanzees fed milk from cows naturally infected with the bovine C-type virus. Cancer Res. 34: 2745-2757.

Moura JW, Stocco dos Santos RC, Dagli ML, D’Angelino JL, et al. (1988). Chromosome aberrations in cattle raised on bracken fern pasture. Experientia 44: 785-788.

Pearce NE, Smith AH, Howard JK, Sheppard RA, et al. (1986). Non-Hodgkin's lymphoma and exposure to phenoxyherbicides, chlorophenols, fencing work, and meat works employment: a case-control study. Br. J. Ind. Med. 43: 75-83.

Recouso RC, Stocco dos Santos RC, Freitas RN, Santos RC, et al. (2003). Clastogenic effect of bracken fern (Pteridium aquilinum $v$. arachnoideum) diet in peripheral lymphocytes of human consumers: preliminary data. Vet. Comp. Oncol. 1: 22-35.

Stocco dos Santos RC, Lindsey CJ, Ferraz OP, Pinto JR, et al. (1998). Bovine papillomavirus transmission and chromosomal aberrations: an experimental model. J. Gen. Virol. 79 ( Pt 9): 2127-2135. 